Chapter 37

\title{
Quality of Life in Patients Undergoing Hemodialysis
}

\author{
Mukadder Mollaoğlu \\ Additional information is available at the end of the chapter \\ http://dx.doi.org/10.5772/45929
}

\section{Introduction}

Quality of life (QoL) is a broad multidimensional concept that usually includes subjective evaluations of both positive and negative aspects of life [1]. What makes it challenging to measure is that, although the term "quality of life"has meaning for nearly everyone and every academic discipline, individuals and groups can define it differently. Philosophers were concerned with the nature of human existence and defined the "good life", ethicists debated the shift in health-care decision-making for the concept of "sanctity of life" to "QoL" and social utility, environmentalists have placed emphasis upon attributes and conditions of the physical and biological environment, economists were concerned with the allocation of resources to achieve alternating goals, psychologists considered human needs and their fulfillment, where as sociologists have advanced a social systems approach in which indicators of QoL are seen as variables in the total system and its subsystems. Physicians focused on health- and illness-related variables and nurses, on keeping with the discipline's holistic approach, took the broadest view in defining life quality, yet because of their frequent preoccupation with the physiological status, they tend to contaminate their operationalization of the concept with disease-specific items [2,3]. And within these disciplines, scientists have defined QoL from different perspectives, such considerations as objective indicators, subjective view, life goals, needs satisfaction, and components of life. WHO defines Quality of Life as individuals perception of their position in life in the context of the culture and value systems in which they live and in relation to their goals, expectations, standards and concerns. It is a broad ranging concept affected in a complex way by the person's physical health, psychological state, level of independence, social relationships, personal beliefs and their relationship to salient features of their environment $[4,5]$.

Although health is one of the important domains of overall quality of life, there are other domains as well-for instance, jobs, housing, schools, the neighborhood. Aspects of culture, values, and spirituality are also key aspects of overall quality of life that add to the complex- 
ity of its measurement [6,7]. Nevertheless, researchers have developed useful techniques that have helped to conceptualize and measure these multiple domains and how they relate to each other [8].

Health-related quality of life (HRQoL) was adapted from the more general and wide-ranging concept 'quality of life'. The concept of HRQoL and its determinants have evolved since the 1980s to encompass those aspects of overall quality of life that can be clearly shown to affect health-either physical or mental [9]. Health-related quality of life is a multi-dimensional concept that includes domains related to physical, mental, emotional and social functioning. It goes beyond direct measures of population health, life expectancy and causes of death, and focuses on the impact health status has on quality of life $[10,11]$.

In the field of medical research, medical sociologist and scientists were concerned with evaluating aspects of life that are affected by disease or treatment for disease, hence, the term health-related QoL were used and included as a criterion for determining the outcome of illness and treatment $[12,13]$.

HRQoL refers to the physical, psychological and social domains of health that are unique to each individual [3].Each of these domains can be measured by the objective assessments of functioning or health status and the subjective perceptions of health. Other valued aspects of life exist that are not generally considered as "health," including income, freedom, and the environment. It has been defined as follows: "HRQoL is defined as the value assigned to duration of life as modified by impairments, functional states, perceptions, and social opportunities that are influenced by disease, injury, treatment, or policy" [8]. Another definition is "HRQoL can be defined as the functional effect of an illness and its consequent therapy upon a patient, as perceived by a patient" [14]. Lehman, Rachuba and Postrado (1995) also suggested that "HRQoL is the optimum level of mental, physical, role, and social functioning, including relationships, and perceptions of health, fitness, life satisfaction, and well-being" [15]. And Bird et al. (2000) defined HRQoL as: "the degree to which valued aspects of a person's life have been influenced, positively or negatively by health and/or health-related interventions such as medical care" [12].

Over the years, consensus has been established that HRQoL is a multidimensional concept. As such, HRQoL is generally divided into 3 domains: physical, social, and psychological (Guyattet al.1993, Testa MA \& Simonson DC, 1996). In the physical domain, perception and observation of normal or disrupted corporal functioning, such as mobility, pain, and nausea, are evaluated. In the social domain, the performance of societal functions is studied; these include activities of daily living and responsibilities in and out of the home, such as those associated with family, friends, and colleagues. In the psychological domain, mental and emotional functioning - for example, patients' concerns, distress, and mood-are examined $[4,6]$.

Briefly, HRQoL refers to the subjective perception of the effect of a disease or its treatment on one's health and overall QoL. It includes physical, psychological, and social dimensions of health as assessed by the patient. It is clearly influenced by the individual's beliefs, life experiences, personality, and expectations [6]. Emphasizing the inherently subjective nature 
of HRQoL is important. The physical dimensions of health (eg, disabilities, impaired physical strength) can be assessed "objectively" through either healthcare personnel or different instruments. These measurements provide information about the patient's "health status" or "functioning." HRQoL, on the other hand, assesses how the presence of the disease's physical symptoms, such as impairment of physical functioning and reduced stamina, affect one's overall well being, life satisfaction, or QoL. This means that two individuals with either similar physical health or equal severity of the disease could have vastly different HRQoL. Evidence accumulated over the last 10-15 years has clearly demonstrated that HRQoL measurements correlate with "objective" measures of physical health and predict traditional "hard outcomes" (ie, hospitalization and mortality) [16]. They also add additional information to the assessment of the overall well being of patients with chronic medical conditions. Clinicians and public health officials have used HRQoL and well-being to measure the effects of chronic illness, treatments, and short- and long-term disabilities. While there are several existing measures of HRQoL and well-being, methodological development in this area is still ongoing.

Recently there has been growing recognition of health-related quality of life as an important indicator of the quality of care for patients with various illnesses. Monitoring patient-reported outcomes (PROs) including self-reported mental and functional health of individuals with chronic disease states is important for assuring optimal disease management and patient satisfaction. The subjective or self-reported state of well being, as it relates to the health condition, also known as "health related quality of life", is a core PRO measure in individuals with End stage renal disease (ESRD). QoL may also serve as a prognostic measure and predictor for such other outcomes as survival.

In order to understand the relationship among the disease, its treatment, and HRQoL, the concept of illness intrusiveness must be understood. Illness intrusiveness was introduced to represent illness-induced disruptions to lifestyles, activities, and interests that compromise QoL [17]. Conceptualized as a facet of the chronic disease experience common across conditions, illness intrusiveness is a fundamental determinant of HRQoL. The central hypothesis is that disease (ie, pain, fatigue, disability) and treatment factors (ie, time required for treatment, untoward side effects) indirectly influence subjective well being and HRQoL through their effects on illness intrusiveness. For example, depriving the individual of the gratifying consequences of psychologically meaningful activities could affect the patient's HRQoL. Psychological and social factors act as moderator variables that influence both the magnitude of illness intrusiveness, which is occasioned by disease and treatment factors, and the degree to which illness intrusiveness compromises QoL [18].

\section{Quality of life of hemodialysis patients}

Over the past few decades, quality of life research endpoints have emerged as valuable research tools in assessing the outcome of therapeutic intervention in chronic diseases [19]. End stage renal disease is one such chronic disease causing a high level of disability in dif- 
ferent domains of the patients' lives, leading to impaired QoL [20,21]. The availability of various renal replacement therapies (RRT) has reduced the severity of symptoms and resulted in longer survival of ESRD patients [22]. Hemodialysis therapy is time-intensive, expensive, and requires fluid and dietary restrictions. Long-term dialysis therapy itself often results in a loss of freedom, dependence on caregivers, disruption of marital, family, and social life, and reduced or loss of financial income [23]. Hemodialysis alters the life style of the patient and family and interferes with their lives. The major areas of life affected by ESRD and its treatment includes employment, eating habits, vacation activities, sense of security, self-esteem, social relationships, and the ability to enjoy life [24]. Due to these reasons, the physical, psychological, socioeconomic, and environmental aspects of life are negatively affected, leading to compromised QoL [25].

Survival of ESRD patients has been largely improved nowadays because of medical progress, advanced technology, and beter patient care. Accumulated data in the recent decade show that health-related quality of life markedly influences dialysis outcomes. Attention thus needs to be focused not only on how long but also on how well ESRD patients live [26]. Compared with the general population, ESRD patients treated with hemodialysis have significantly impaired HRQoL [27].

Evaluation of HRQoL in patients with chronic diseases is becoming very important. HRQoL assessment helps to plan the individual strategy of treatment, to determine the efficacy of medical intervention, and to evaluate the quality of medical care. In comparison with HRQoL of the general population, it provides the opportunity to evaluate the psychological burden of chronic disease, and the effect of specific treatment [28].Some studies have shown international differences in HRQoL of ESRD patients treated with hemodialysis [29, 30].

An increasing number of professionals feel that HRQoL assessment is essential to evaluating quality and effectiveness of ESRD patient care, comparing alternative treatments and RRT modalities, improving clinical outcomes, facilitating complex rehabilitation of ESRD patients, and enhancing patient satisfaction. Several authors have suggested that regular HRQoL monitoring become part of regular ESRD patient assessment and incorporated into the continuous quality assurance and quality improvement systems [31,32].

ESRD is a life-threatening disease that leads to numerous and severe symptoms and complications. These severe comorbid conditions will have a major impact on the affected patients' HRQoL. RRTs are able to alleviate, but they are very intrusive and cure neither the disease nor its symptoms. Patients suffering from ESRD need RRTs to survive, but they also expect to achieve a certain level of well being. In industrialized countries achieving survival is not enough for a treatment to be considered "successful" unless it also yields an appreciable gain in HRQoL $[33,34]$. Thus, the results of studies suggest that the QoL of hemodialysis patients is considerably impaired compared to that of the healthy subjects, especially with respect to the physical, psychological and social relationship domains [35,36]. In a previous DOPPS study, lower scores in several measures of HRQoL, particularly PCS, were found to be strongly associated with higher risk of death in Japan, Europe, and the United States [16]. Other studies have shown that patients on hemodialysis have a poor health-related quality of life (HRQoL) and present with complications such as depression, malnutrition, and in- 
flammation. Many of them suffer from impaired cognitive functioning such as memory loss and abnormally low concentration, as well as other unhealthy physical, mental, and social aspects of life that can, and do, affect even the simplest activities of daily life [37,38]. On the other hand, many researchers emphasize that an improvement in HRQoL reduces the complications associated with this disease, or at least makes them more tolerable [39]. Therefore, it is useful to determine the level of renal function related to the decreasing point of HRQoL for the adequate intervention to enhance HRQoL in time. Improving QoL and other PROs in the dialysis patient population has evolved as a goal for renal replacement therapy.

\section{Factors associated with health-related quality of life in patients under going hemodialysis}

End-stage renal disease patients undergoing hemodialysis (HD) has a considerable impact on the functional status and health-related QoL perceived by the patient as it is accompanied by symptoms that affect daily life [40].Over the years, several studies have assessed HRQoL in different ESRD populations. These reports reveal numerous sociodemographic, clinical, and psychosocial factors that are associated with impaired HRQoL.

Sociodemographic factors: It has been repeatedly demonstrated patients undergoing hemodialysis that female patients consistently report worse HRQoL than men [40,41]. Women had lower QoL scores than men, as already reported by the studies [42,43]; this may be explained by women's multiple domestic tasks and responsibilities that, unlike men, they cannot circumvent [44]. Also, one potential explanation may be the more negative disease perception and the increased prevalence of depression in women. Moreno et al.,(1996) in their multicenter cross-sectional study [36], and Sesso et al., (2003) in their prospective cohort study, also found that higher socioeconomic level was significantly related to better QoL [45]. A lower social status, characterized by lower education, worse financial situation, or lack of employment, has also been consistently associated with impaired QoL $[41,46]$. This association is important, as vocational and educational rehabilitation could substantially improve HRQoL. The association of age with HRQoL is quite complex and illustrates the complexity of the QoLconcept.Some studies conducted in different countries also demonstrated that age was strongly inversely associated with the physical domain scores [25].As age increases in the elderly, physical function of the body decreases [46-48]. The subjective QoL for elderly patients, however, varies depending on their expectations and beliefs. It could be surprisingly good compared to their younger counterparts [49].

Clinical factors: Several clinical factors are strongly associated with HRQoL in hemodialysis patients. The underlying kidney disease leading to renal failure, the presence and severity of diabetes [50,51] and comorbid conditions in general $[49,52]$ and congestive heart failure in particular predict impaired QoL [53]. Anemia is highly prevalent in patients undergoing HD and is associated with adverse clinical outcomes and diminished HRQoL [54-59]. The most prominent symptoms of anemia are fatigue, dyspnea, and diminished sense of well-being. Less common symptoms include difficulty concentrating, dizziness, sleep disorders, cold in- 
tolerance, and headaches [60]. Walters et al. (2002) assessed health-related QoL, depressive symptoms, anemia, and malnutrition at hemodialysis initiation and found that $56 \%$ of the sample group (422) had a hemoglobin levels less than 10g/dl [38]. Chronic inflammation, presence of malnutrition, and different medications' side effects have been reported to predict worse HRQoL [31]. However, it is important to note that the different comorbidity indices are used to measure comorbid burdens, and clinical and sociodemographic factors only explains a fraction of HRQoL variability.

Duration of dialysis plays an important role affecting QoL in dialysis patients. According to Vasilieva (2006), in linear regression analysis, duration of dialysis was a significant independent predictors of the low physical component score (PCS) in hemodialysis patients [61]. A similar observation was made by Anees et al. (2011); duration of dialysis had a reverse correlation with QoL. As duration of dialysis increases, QoL of dialysis patients deteriorates [62]. In another study, QoL was better in hemodialysis patients with a duration less than 8 months than patients with a dialysis duration more than 8 months [63].

Psychological/psychosocial factors: Several psychosocial factors have also been shown to strongly predict HRQoL scores. The expanding awareness about objective parameters and their impact on HRQoL is complemented by few studies on subjective symptoms and their influence on HRQoL in CKD [64-66]. The symptom burden that these patients struggle with include, fatigue $[59,64,65]$, cognitive difficulties [59,64], sleep disturbances $[34,59,64,67]$, sexual dysfunction $[59,64]$, pain and depression $[59,64,65]$, most of which are interlinked $[65,67$. While larger studies on pre-dialysis ESRD patients are lacking, existing ones confirm the negative impact of these symptoms on HRQoL $[34,59,66]$.

In relation to psychological status, the a study by Mollaoglu (2004) indicated that two third of ESRD patients in Turkey had depression and found an association between depressed mood and health-related QoL [63]. The higher depression scores the lower health-related QoL scores. She explained that as a direct influence of chronic renal insufficiency on healthrelated QoL. Another study indicated that the mental health was significantly higher for patients treated in the United States than in Europe [68]. In another study by Jofre, LopezGomez \&Valderrabano (2000) who reviewed the factors affecting the QoL of renal failure patients, they found that the prevalence of depression is within $70 \%$ in the dialysis population using Beck Depression Inventory (BDI), they also indicated that depression has a significant impact on the perception of QoL [40]. Anxiety is another psychological response to hemodialysis patients and is related to the awareness of one's illness and the sense of dependency on the machine. Patients are concerned about the unpredictability of the illness and the disruption of their lives, they are chronically ill and fear dying [24].

Body image is also affected by dialysis treatment, making patients feel different, unattractive, and ill at ease within their own bodies. Access surgery often results in multiple scarring, involving the arms and chest. A fistula which is regarded as "very good" by dialysis staff can be seen as a horrible disfigurement by the patient, who may try to conceal it from friends and the curious stares of strangers. Many feel embarrassed in front of their partners and feel that nobody could find them attractive anymore $[63,64]$. 
Anxiety, loss of control, body image and sexual problems, social support, and unemployment are all factors that strongly influence QoL in hemodialysis patients. The utmost significance of these factors is further underlined by the fact that many of them are modifiable. Unfortunately, little attention is given to assess the potentially modifiable psychosocial stressors in hemodialysis patients.

Sleep disorders are highly prevalent in patients with renal impairment. The most frequent sleep disorders, such as restless legs syndrome, periodic leg movements in sleep, insomnia, and obstructive sleep apnea, are associated with significantly impaired HRQoL in patients with moderate renal failure not yet requiring RRTs as well as in patients on hemodialysis $[17,32]$

\section{Assessment of health-related quality of life in patientsundergoing hemodialysis}

Health-related QoL assessment, as a supplement to more objective clinical indicators, is becoming more topical in view of the increasing questioning of the effectiveness and appropriateness of many existing medical treatments and methods of organizing health services [2,4]. The US Centers for Disease Control and Prevention CDC (1993) suggested that: measuring health-related QoL can help determine the burden of preventable diseases, injuries, and disabilities, and it can provide valuable new insights into the relationships between health-related QoL and risk factors. Measuring health-related QoL will help monitor progress in achieving the notions' health objectives. Analysis of health-related QoL surveillance data can identify subgroups with relatively poor perceived health and help to guide interventions to improve their situations and avert more serious consequences [69].

In the field of nephrology, the evaluation of health-related QoL involves determining the efficiency and effectiveness of the different forms of renal replacement therapy (e.g. HD and peritoneal dialysis), evaluating the efficiency and effectiveness of the different types of other treatments applied to patients with ESRD (e.g., recombinant human erythropoietin therapy) and follow-up of the evolution of individual renal patients [43].

Different generic disease-specific instruments and domain-specific instruments have been used for assessing the QoL in patients undergoing hemodialysis (Germin-Petrovic et al. 2011).

\subsection{Generic instruments}

Measures which implicitly or explicitly aim to tap health-related QoL. They encompass the dimensions of physical, mental and social health [69]. These instruments are intended for general use, irrespective for the illness or condition of the patient. These generic questionnaires may often be applicable to healthy people, too [13]. The Sickness Impact Profile, the Medical Outcome Study 36-Item Short Form, and the Nottingham Health Profile are examples of the generic instruments. 


\subsubsection{Medical outcome study 36-item short Form (SF-36)}

The SF-36 developed by Ware et al. in 1993 evaluates general health status, it is designed to provide assessments involving generic health concepts that are not specific to any age, disease or treatment groups. Emphasis is placed upon physical, social, and emotional functioning. It can be either self-assessed or administered by a trained interviewer. As the name implies, there are 36 questions addressing physical health and mental health [13].

\subsubsection{The Nottingham Health Profile (NHP)}

The Nottingham Health Profile (NHP) was developed to be used in epidemiological studies of health and disease [70]. It consists of two parts. Part I contains 38 yes/no items in 6 dimensions: pain, physical mobility, emotional reactions, energy, social isolation and sleep. Part II contains 7 general yes/no questions concerning daily living problems. The two parts may be used independently. Part I is scored using weighted values which give a range of possible scores from zero (no problems at all) to 100 (presence of all problems within a dimension).

\subsubsection{Sickness impact profile}

A 136-item self- or interviewer-administered, behaviorally-based, health status questionnaire. Everyday activities in 12 categories (sleep and rest, emotional behavior, body care and movement, home management, mobility, social interaction, ambulation, alertness behavior, communication, work, recreation and pastimes, and eating) are measured. Respondents endorse items that describe themselves and are related to their health. The SIP is scored according to the number and type of items endorsed. Scoring can be done at the level of categories and dimensions as well as at the total SIP level. It may be either interviewer- or self-administered [13].

\subsubsection{Disease-specific instruments}

\subsubsection{Quality of life index-D (QLI-D)}

The Quality of Life Index (QLI) was developed in the USA during the 1980s as a measure of morbidity for application in both normal and unwell populations [71]. The original instrument, with the addition of six dialysis-specific items, was developed and tested in patients receiving haemodialysis [71]; factor analysis confirmed instrument construction. The instrument comprises two sections assessing respondent satisfaction and relative importance of each domain, respectively. Each section has 32 items, with eight items per domain. Six-point ordinal response scales range from 'very dissatisfied' or 'very unimportant' (1), to 'very satisfied' or 'very important' (6). Scoring is complicated and the developers recommend a computer programme. In summary, importance scores are used to weight satisfaction scores; index or domain scores range from 0 to 30, where higher scores indicate better quality of life.

Regarding evidence in relation to kidney disease, reliability was supported for the QLI in a one month time interval for dialysis patients. High internal consistency was also reported 
in a small study by [71]and reproduced in a further study with a larger sample of patients [71]. Additional items for haemodialysis patients relating to treatment were added to each section (Satisfaction with various domains and Importance of the domain to the individual) in Ferrans and Powers 1985). Items were endorsed by patients receiving haemodialysis. A transplant version is also available which included two items relating to the potential for a successful transplant. This is for patients receiving haemodialysis and on the transplant list.

A four factor structure was supported in Ferrans and Powers (1985) of Health and functioning, socioeconomic, psychological/spiritual and family. A high order factor was revealed representing Quality of Life. Moderate correlation of QLI-D scores with a life satisfaction questionnaire has been reported [71]. Further convergent validity is supported for each domain and life satisfaction, with higher correlations for the Psychological/spiritual domain. Moderate correlation was reported between scores from the QLI-D and other patient-reported measures of symptoms and psychological adjustment to disease. Moderate correlation of scores has been reported between QLI-D and symptoms.

A larger population was recruited in another study by the developers [13]. This included 349 patients from a haemodialysis unit and questionnaires mailed to patients. $20 \%$ of patients had missing values greater than $15 \%$ and overall computable responses were available from $46 \%$ of participants invited. A $46 \%$ response rate was obtained to postal administration of the questionnaire [13].

\subsubsection{Kidney Disease Quality of Life Short Form (KDQOL-SF)}

The KDQOL-SF includes both general measures and measures specific to patients with kidney disease. The general measures were based on questions from the 36-item Short-Form Health Survey (SF-36), developed by Ware and Sherbourne [73]. Previous data support the use of the SF-36 and the KDQOL-SF as research instruments to HRQoL [74]. The internal consistency and reliability are similar among translations of the SF-36 and the KDQOL-SF $[9,68]$. Patient responses to the SF-36 questions were used to determine scores for the mental component summary (MCS) and the physical component summary (PCS). The scales for MCS and PCS are derived from eight different subscales: physical functioning role (physical, bodily pain, general health, and vitality) and social functioning role (emotional, and mental health).

The KDQOL-SF includes questions that supplement the SF-36. These additional questions were designed to assess the particular health-related concerns of individuals with kidney diseases and ESRD patients treated by dialysis [74]. The kidney disease component summary (KDCS) score, which corresponds to the MCS and PCS of the SF-36, is derived from 11 subscales: symptoms/problems, effects of kidney disease on daily life, burden of kidney disease, work status, cognitive function, quality of social interaction, sexual function, sleep, social support, dialysis staff encouragement, and patient satisfaction.

The SF-36 and kidney disease-targeted portions of the questionnaire were scored according to the manual by Ware et al (1993) and the KDQOL scoring manual (Hays et al.1994) [74]. 
On all scales, the possible scores range from 0 to 100; higher scores indicate more or better functioning, or better quality of life. The summary scales have the same interpretation, but do not span the entire 0 to 100 range.

\subsubsection{Kidney Disease Questionnaire (KDQ)}

This 26 itemed questionnaire was developed in Canada with the involvement of patients receiving haemodialysis and empirically by factor analysis. Five domains included are: Physical symptoms (6 individualised symptoms identified by the patient); Fatigue: 6); Depression (5); Relationships (6); Frustration (3). Responses are scored in a 7 point Likert scale during the last 2 weeks. It is reported to take 10 to 15 minutes to complete.

Reproducibility is supported with ICCs above 0.80 for all domains. Construct validity is reported with moderate correlations with analogous domains using the SIP. Trial data [76], provide support for responsiveness with significant improvement in scores for patients receiving treatment for anaemia which was consistent with score changes on the SIP.

\subsubsection{Renal Quality of Life Profile (RQLP)}

The Renal Quality of Life profile (RQLP) is a 43 itemed questionnaire with a 5 point Likert scale for responses. Five dimensions include: Eating and drinking, Physical activities, Leisure time, Psychosocial activities and Impact of treatment. It was developed adopting a comprehensive methodology involving patients and clinicians in the UK [77].

Principal component factor analysis supported the five dimensions. A high response rate is reported in Barton et al., [78].The RQLP scores were responsive to change in a trial of pharmacy care compared to standard care for patients receiving HD. Effect sizes were moderate [78]. Moderate correlation is reported between the RQLP and SF-36 dimensions which were similar in construct.

\subsubsection{CHOICE Health Experience Questionnaire (CHEQ)}

The Choices for Healthy Outcomes in Caring for ESRD (CHOICE) study was designed to evaluate the effectiveness of alternative dialysis prescription. As part of the CHOICE study, the CHEQ as patient-reported HRQoL instrument was developed to specifically complement the SF-36; be sensitive to dialysis treatment modalities and regimes; and be useful for longitudinal evaluation. A comprehensive, patientcentred approach was used during development. Items were derived from interviews with patients; literature; and clinicians' expertise [79]. The questionnaire has 83 items addressing 21 domains: the 8 domains of the SF-36, 8 additional generic domains (cognitive functioning, sexual functioning, sleep, work, recreation, travel, finances, and general quality of life); and 5 ESRD-specific domains (diet, freedom, body image, dialysis access. The original study byWu and colleagues (2001) provided some evidence for the reliability and validity of the scales. Adequate internal consistency is reported for most domains in Wu et al. (2001) [79]. 


\subsubsection{Renal dependantindividualised quality of life questionnaire}

This instrument was developed out of an instrument used in relation to diabetes, the Audit of Diabetes Dependent Quality of Life (ADDQoL) diabetes-specific individualized quality of life questionnaire. From a small study with patients in eight U.K. renal clinics each of the 13 ADDQoL items were found relevant and important for renal patients. Additional items were also identified by patients including physical appearance, dependency, freedom, restrictions of fluid intake, and societal prejudice [80]. No psychometric data for the new instrument were reported. No further studies using the instrument were identified.

\subsection{Domain-specific instruments}

\subsubsection{Barthel Index of disability (BI)}

The BI was developed in 1965 (Barthel, 1965)and later modified by Granger and coworkers (1979) as a scoring technique that measures the patient's performance in 10 activities of daily life $[81,82]$. The BI is considered a reliable disability scale for stroke patients. The items can be divided into a group that is related to self-care (feeding, grooming, bathing, dressing, bowel and bladder care, and toilet use) and a group related to mobility (ambulation, transfers, and stair climbing). The maximal score is 100 if 5-point increments are used, indicating that the patient is fully independent in physical functioning. The lowest score is 0 , representing a totally dependent bedridden state. The MRS measures independence rather than performance of specific tasks The BI examines the ability to perform normal or expected activities [13]. In this way, mental as well as physical adaptations to the neurological deficits are incorporated. The scale consists of 6 grades, from 0 to 5, with 0 corresponding to no symptoms and 5 corresponding to severe disability.

\subsubsection{McGill Pain Questionnaire (MPQ)}

The McGill Pain Questionnaire, also known as McGill pain index, is a scale of rating pain developed at McGill University by Melzack and Torgerson [83].

To use the questionnaire, circle the words that describe your pain but do not circle more than one word in a group. Then when you have that done, go back and circle the three words in groups 1-10 that most convey your pain response. Pick the two words in groups 11-15 that do the same thing. Then pick one word in group 16. Finally, pick 1 word in groups 17-20. At the end you should have seven words that you can take to your doctor that will help describe both the quality of your pain and the intensity of it [13].

\section{Improving health-related quality of life in patientsundergoing hemodialysis}

There is growing recognition of health-related QoL issues in ESRD patients undergoing hemodialysis. Considerable progress has been made in the treatment and health intervention 
of chronic kidney disease, however, health-related QoL continues to be a significant problem for patients receiving hemodialysis [31,39]. Hemodialysis patients are subjected to multiple physiological and psychological stressors and may be threatened with many potential losses and life style changes as they experience problems with disease-specific symptoms. The combination of a decrease in energy, the unavoidable emergence of socioeconomic problems, and emotional reactions compounds the stress facing the patient [38,45]. The initiation of long-term dialysis treatment increases survival, but health-related QoL remains impaired. Therefore, researchers and clinicians generally agree that health-related QoL, its determinants and treatment options that may preserve subjective well-being merit continued investigation $[62,63]$.

Health care workers should understand the health-related QoL of patients undergoing hemodialysis. The rich information collected can help health professionals to determine which patients may be at risk for diminished health-related QoL. It has direct consequences for clinical decision-making, rehabilitation and management of individual patients [65,72]. Draper (1992) stated that health professionals, in their decisions and actions, can influence their patient's QoL [84]. Additionally, they will be interested in promoting these conditions, which enhance life's quality, and eliminating those that impair it. Health professionals working in hemodialysis units can direct resources to areas where improvement may be required. Patients can then have a greater chance of leading a fulfilling life. All these factors can positively influence the health-related QoL of patients, and directly benefit the family as well $[62,63,65]$. This could be accomplished through health education and promotion of awareness about the disease, treatment options, complications and self-care activities. Counseling, on the other hand, is an important intervention that health professionals - with appropriate training - can provide. Referral of patients to the appropriate person according to their needs could be provided by an ordinary health professional who cares for the patient. Finally, health professionals can develop and implement rehabilitation programs for ESRD patients undergoing hemodialysis to assist them lead a productive life.

ESRD has a profound effect on HRQoL with the most prominent areas of difficulty being the physical domains. Hemodynamic instability is a major problem observed in hemodialysis patients and thus managed carefully. Anemia management in ESRD patients is a challenge for the health care team. The use of erythropoietin-stimulating agents (ESAs) has become a routine practice in hemodialysis patients in an effort to correct anemiaand improve HRQoL. Nonetheless, two simultaneously published trials $[85,86]$ raised concerns regarding the optimal hemoglobin target levels. Druekeet al. (2006), the CREATE investigators, reported significant increment in HRQoL with higher hemoglobin levels whereas Singh et al. (2006), the CHOIR investigators, reported no difference in the HRQoL between the low and high hemoglobin arms after EPO therapy [86]. Additionally, normalization of hematocrit was shown to be associated with adverse cardiovascular outcomes in the CHOIR trial (2006). In consideration of the conflicting results of these publications, the National Kidney Foundation Kidney Disease Outcomes Quality Initiative (NKF KDOQI) published revised guidelines for the management of anemia in CKD patients [87], which were further reviewed at the Kidney Disease: Improving Global Outcomes (KDIGO) international conference. Members voiced a 
general consensus on maintaining target hemoglobin levels in the range of 11.0 to $12.0 \mathrm{~g} / \mathrm{dL}$ (KDOQI 2007) and acknowledged the potential for harm associated with levels higher than $13.0 \mathrm{~g} / \mathrm{dL}$ [87].

Considering the dramatically increasing prevalence of ESRD, the risk of progression of ESRD with hypertension and the significant impact of the disease on HRQoL, improving HRQoL is emerging as one of the therapeutic goals in hypertensive individuals. High blood pressure is managed by an appropriate choice of antihypertensive medications to have a target blood pressure of $125 / 75 \mathrm{mmHg}$. Cardiovascular risk factors are minimized as ESRD patients are at increased risk for coronary heart disease over the standard risk factors, prevention includes frequent monitoring of plasma lipid levels, diet control, and pharmacologic treatment of specific hyperlipidemias [87].

The increasing prevalence of ESRD in the older population and the poor prognosis and impaired HRQoL associated with frailty warrant early identification of high-risk patients. Suggested management strategies to prevent further deterioration include exercise training and correction of malnutrition, anemia, depression and hormonal imbalances. Growth hormone supplementation in elderly dialysis patients has been shown to improve muscle performance and HRQoL [88], but whether this approach would be helpful in patients with non-dialysis dependent CKD remains to be established.

Among the psychological stressors of ESRD patients undergoing HD, depression and anxiety are the most common problems encountered. The onset of ESRD and HD impacts significantly one's functional state and health-related QoL, it causes major alterations in the lifestyle of most patients, who may encounter frustration in all areas of life, this frustration causes depression which is known to be strongly associated to decreased health-related QoL. This is illustrated in Walters et al. (2002) study that assessed health-related QoL, depressive symptoms, anemia and malnutrition at HD initiation and found that HD patients who screened positive for depression ( $45 \%$ of the sample) scored lower on health-related QoL scale [38]. Anxiety, on the other hand, is detected in HD patients, and is caused by unstable health status leading to fears from worsening health condition, disturbed social relations, unemployment and consequent economic alterations, and even death. A study by White and Grenyer (1999) that aimed at investigating the impact of dialysis on both the patient and their partner found that dialysis patients had anxiety as they expressed uncertainty related to health instability within a progressively debilitating disease state and frequent interruptions of acute illness episodes [89].

Socioeconomic status is also altered in ESRD patients undergoing HD as chronic dialysis imposes a considerable burden on patients and families [17,38], the relationships of the patients with family members is altered as there is role reversal, with the assumption of added responsibilities by the spouse, resulting in a loss of authority for the patients [63]. Social isolation and decreased social interactions is observed in HD patients and this is caused by their health status and the treatment schedules. Another alteration of lifestyle includes the probable loss of financial security resulting from lower productivity and income, and possible unemployment. All the above factors are strongly related to health-related QoL of HD patients. Parkerson and Gutman, (2000) assessed health-related QoL of 103 ESRD patients on HD, 
and found that patients living with family reported more social support and better healthrelated QoL, general health, emotional well-being, social health and quality of social interactions than other patients [89].

The development and evaluation of effective interventions to reduce psychological distress, improve QoL and enhance social intimacy are of clinical and scientific importance to HD patients, their family members and healthcare providers. Tsayand Lee, (2005) randomized patients with end-stage renal disease to a cognitive-behavioural coping skills and stress management training programme or standard care (primarily education) [91]. Cognitive-behavioural treatment reduced symptoms of stress and depression, and improved QoL, compared with a standard care condition. Chang et al. (2004) combined education, vocational rehabilitation and social support enhancement, and found significant QoL improvements in ESRD patients [92]. Gross et al. (2004) used a mindfulness-based stress reduction programme in ESRD patients to reduce depression and anxiety, although no QoL improvements were found. Quality of life therapy (QoLT) is the only cognitive-behavioural treatment that targets happiness and life satisfaction in multiple life domains (e.g. relationships, enjoyable activities, self-esteem, etc.) with a specific goal of improving overall QoL [93]. This is important because the World Health Organization has emphasized the importance of a patient's subjective perception of life in the context of his or her value systems, goals, expectations and standards.

\section{Conclusion}

Although advances in dialysis treatment have contributed to improved survival of patients with end-stage renal disease,such individuals particularly those treated by hemodialysis, health-related quality of life is much lower for those patients than for the general population. Impaired health-related QoL, dependence on others, and poor rehabilitation all contribute to physical and emotional disabilities that may persist even in well-dialyzed ESRD patients. Chronic HD patients are subjected to multiple physiological and psychological stressors and may be threatened by many potential losses and lifestyle changes. Analysis of health-related QoL surveillance data can identify subgroups with relatively poor perceived health and help to guide interventions to improve their situations and avert more serious consequences. Developments of HD technology, treatment of comorbidities, continuous patients' education, social and psychological support may improve the HRQoL in these patients.

\section{Author details}

Mukadder Mollaoğlu

Cumhuriyet University, Health Sciences Faculty, Sivas, Turkey 


\section{References}

[1] Centra, L. New information about health-related quality of life. NACCHO News; (1998).

[2] Anderson KL, Burckhardt CS.Conceptualization and measurement of quality of life as an outcome variable for health care intervention and research. J AdvNurs (1999). , 29(2), 298-306.

[3] Bubloz, M., Eicher, J. B., Evers, S. J., Sontag, A., human, ecological., approach, to., quality, of., life, Conceptual., framework, , results, of. a., \& preliminary, study. Soc Indic Res. (1980).

[4] Guyatt GH, Feeny DH, Patrick DL.Measuring health-related quality of life. Ann Intern Med. (1993). , 118, 622-629.

[5] The WHOQOL Group. World Health Organization.Quality of Life Assessment ( theWHOQOL) : position paper from the World Health Organization. SocSci Med. (1995).

[6] Testa MA, Simonson DC.Assessment of quality-of-life outcomes. New Eng J Med. (1996). , 334(13), 835-840.

[7] Samman, E., Psychological, , Subjective, Well-being. A., Proposal, for., Internationally, Comparable., \& Indicators, . Oxford Development Studies (2007). , 35(4), 459-486.

[8] Patrick, D. L., \& Erickson, P. Applications of Health Status Assessment to Health Policy. In: Spilker B, ed. Quality of Life and Pharmacoeconomics in Clinical Trials. Second ed. Philadelphia, Lippincott-Raven Publishers, (1996). , 717-727.

[9] Gandek, B., Sinclair, S. J., Kosinski, M., et al. Psychometric evaluation of the SF-36 health survey in medicare managed care. Health Care Financ Rev. (2004). , 25(4), $5-25$.

[10] Mc Horney, . Health status assessment methods for adults: past accomplishments and future challenges. Annu Rev Publ Health. (1999). , 20, 309-35.

[11] Selim, A. J., Rogers, W., Fleishman, J. A., et al., \& Updated, U. S. population standard for the Veterans RAND 12-item Health Survey (VR-12). Qual Life Res. (2009). , 18(1), 43-52.

[12] Bird, Conrad. P., \& Fremont, A. M. Handbook of medical sociology. th ed. New Jersey: USA: Prentice-Hall, Inc; (2000).

[13] Fayers, P. M., \& Machin, D. Quality of life assessment, analysis, and interpretation. Chichester: England: John Wiley \& Sons Ltd: (2000).

[14] Schipper, H., Clinch, J. J., \& Olweny, C. L. M. Quality of Life Studies: Definitions and Conceptual Issues. In:Spilker B, ed. Quality of Life and Pharmacoeconomics in Clinical Trials. Second ed. Philadelphia: Lippincott-Raven Publishers; (1996). , 11-23. 
[15] Lehman AF, Rachuba LT, Postrado LT. (1995). Demographic influences on quality of life among persons with chronic mental illness. Evaluation and Program Planning, 18, 156-164.

[16] Mapes, D. L., Lopes, Satayathum. S., et al. Health-related quality of life as a predictor of mortality and hospitalization: The Dialysis Outcomes and Practice Patterns Study (DOPPS). Kidney Int (2003).

[17] Devins, G. M., Mann, J., Mandin, H., et al. Psychosocial predictors of survival in endstage renal disease. J NervMent Dis. (1990). , 178(2), 127-133.

[18] Devins, G. M., Beanlands, H., Mandin, H., \& Paul, L. C. Psychosocial impact of illness intrusiveness moderated by self-concept and age in end-stage renal disease. Health Psychol. (1997). , 16(6), 529-538.

[19] Kaufman SE.The increasing importance of quality of life research. Clin Res. (2001). , $1,18-22$.

[20] Edgell, E. T., Coons, S. J., Carter, W. B., et, al. A., review, of., Health-Related, qualityof-life., measures, used., in, end., stage, renal., \& disease, Clin. ClinTher. (1996). , 18, 887-938.

[21] Fox, E., Peace, K., Neale, T. J., et al. Quality of life for patients with end stage renal failureRen Fail. (1991). , 13, 31-5.

[22] Hudson JQ, Johnson CA. Chronic kidney disease. In: Koda Kimble MA, et al., editors. Applied therapeutics. 8th ed. Philadelphia: Lippincott Williams and Wilkins;. (2004). 32-31.

[23] Lin CC, Lee BO, Hicks FD.The phenomenology of deciding about hemodialysis among taiwanese. West J Nurs Res. (2005). , 27, 915-29.

[24] Smeltzer, S. C., \& Bare, B. Brunner \&Suddarth's textbook of medical-surgical nursing. th ed. Philadelphia: USA: Lippincott Williams \& Wilkins: (2004).

[25] Blake, C., Codd, M. B., Cassidy, A., et al., Physical, function., employment, , quality, of., life, in., end-stage, renal., \& disease, . J Nephrol. (2000). , 13, 142-9.

[26] Lopes-Gresham, Bragg., Satayathum, J. L., et, S., \& al, . Worldwide Dialysis Outcomes and Practice Patterns Study Committee. Health-related quality of life and associated outcomes among hemodialysis patients of different ethnicities in the United States: the Dialysis Outcomes and Practice Patterns Study (DOPPS). Am J Kidney Dis. (2003). , 41, 605-615.

[27] Molsted, S., Aadahl, M., Schou, L., \& Eidemak, I. Self-rated health and employment status in chronic hemodialysis patients. Scand J UrolNephrol (2004). , 38, 174-8.

[28] Mapes, D. L., Bragg-Gresham, J. L., Bommer, J., et al. Health-related quality of life in the Dialysis Outcomes and Practice Patterns Study (DOPPS). Am J Kidney Dis. (2004). suppl 2):, 54 EOF-60 EOF. 
[29] Saran, R., Bragg-Gresham, J. L., Rayner, H. C., et al., Nonadherence, in., hemodialysis, Associations., with, mortality., hospitalization, , practice, patterns., \& in, the. D. O. P. P. S. Kidney Int (2003).

[30] Takai, I., Fukuhara, S., Nakai, S., et al. Effect of creatinine generation rate on the relationship between hemodialysis prescription and health-related quality of life. J Artif Organs (2002).

[31] Kalantar-Zadeh, K., \& Unruh, M. Health related quality of life in patients with chronic kidney disease. IntUrolNephrol. (2005). , 37(2), 367-378.

[32] Unruh ML, Weisbord SD, Kimmel PL.Health-related quality of life in nephrology research and clinical practice. Semin Dial. (2005). , 18(2), 82-90.

[33] Birmele, B., Francois, M., Pengloan, J., et al. Death after withdrawal from dialysis: the most common cause of death in a French dialysis population. Nephrol Dial Transplant. (2004). , 19(3), 686-691.

[34] Cohen LM, Germain MJ.The psychiatric landscape of withdrawal. Semin Dial. (2005). , 18(2), 147-153.

[35] DeOreo PB. Hemodialysis patient- Assessed functional health status predicts continued survival, hospitalization and dialysis- attendance compliance.Am J Kidney Dis. (1997). , 30, 204-12.

[36] Moreno, F., Lopez, Gomez. J. M., Sanz-Guajardo, D., et al. Quality of life in dialysis patients: a Spanish multicenter study. Spanish Cooperative Renal Patients Quality of life study group: Quality of life in dialysis patients. A Spanish multicentre study. Nephrol Dial Transplant. (1996). , 11, 125-129.

[37] Vos, P. F., Zilch, O., Jennekens-Schinkel, A., et al. Effect of short daily home haemodialysis on quality of life, cognitive functioning and the electroencephalogram. Nephrol Dial Transplant. (2006). , 21, 2529-35.

[38] Walters BA, Hays RD, Spritzer KL, et al.Health-related quality of life, depressive symptoms, anemia, and malnutrition at hemodialysis initiation. Am J Kidney Dis. (2002). , 40, 1185-94.

[39] Mau LW, Chiu HC, Chang PY, et al.Health-related quality of life in Taiwanese dialysis patients: effects of dialysis modality. Kaohsiung J Med Sci.(2008). , 24, 453-60.

[40] Jofre, R., Lopez-Gomez, J. M., \& Valderrabano, F. Quality of life for patient groups. Kidney Int. (2000). , 57, 121-130.

[41] Rocco MV, Gassman JJ, Wang SR, et al.Cross-sectional study of quality of life and symptoms in chronic renal disease patients: the Modification of Diet in Renal Disease Study. Am J Kidney Dis. (1997). , 29(6), 888-896.

[42] Harris LE, Luft FC, Rudy DW et al.Clinical correlates of functional status in patients with chronic renal insufficiency. Am J Kidney Dis. (1993). , 21, 161-166. 
[43] Rebollo, P., Ortega, F., Baltar, J. M., et al. Health-related quality of life (HRQoL) in end stage renal disease (ESRD) patients over 65 years. GeriatrNephrol Urol. (1998). , $8,85-94$.

[44] Turner-Musa, J., Leidner, D., Simmens, S., et al. Family structure and patient survival in an African-American end-stage renal disease population: a preliminary investigation. SocSciMed. (1999). , 48, 1333-1340.

[45] Sesso, R., Rodrigues-Neto, J. F., \& Ferraz, M. B. Impact of socioeconomic status on the quality of life of ESRD patients. Am J Kidney Dis. (2003). , 41, 186-95.

[46] Simmons, R. G., \& Abress, L. Quality-of-life issues for end-stage renal disease patients. Am J Kidney Dis. (1990). , 15(3), 201-208.

[47] Kimmel PL, Peterson RA, Weihs KL, et al.Aspects of quality of life in hemodialysis patients. Am SocNephrol. (1995). , 6(5), 1418-1426.

[48] Tseng HM, Lu JFR, Tsai YJ.Assessment of health-related quality of life in Taiwan (II): norming and validation of SF-36 Taiwan version. Taiwan J Public Health (2003). , 22, 512-8.

[49] Barotfi, S., Molnar, M. Z., Almasi, C., et al. Validation of the Kidney Disease Quality of Life-Short Form questionnaire in kidney transplant patients. J Psychosom Res. (2006). , 60(5), 495-504.

[50] Kızılışık AT, Feurer ID, VanBuren DH, et al.Effects of diabetes and cadaveric organs on functional performance and health-related quality of life after kidney transplantation. Am J Surg. (2003). , 186(5), 535-539.

[51] Sorensen, V. R., Mathiesen, E. R., Watt, T., et al. (2007). Diabetic patients treated with dialysis: complications and quality of life. Diabetologia, 50(11), 2254-2262.

[52] Van Manen JG, Korevaar JC, Dekker FW, et al.Adjustment for comorbidity in studies on health status in ESRD patients: which comorbidity index to use? J Am SocNephrol. (2003). , 14(2), 478-485.

[53] Silverberg, Wexler. D., Blum, M., et al. Effects of treatment with epoetin beta on outcomes in patients with anaemia and chronic heart failure. Kidney Blood Press Res. (2005). , 28(1), 41-47.

[54] Dowling TC. Prevalence, etiology, and consequences of anemia and clinical and economic benefits of anemia correction in patients with chronic kidney disease: an overview.Am J Health Syst Pharm. (2007). Jul 1;64(13 Suppl 8):S, 3-7.

[55] Kimel, M., Leidy, N. K., Mannix, S., et al. Does epoetinalfa improve health-related quality of life in chronically ill patients with anemia? Summary of trials of cancer, HIV/AIDS, and chronic kidney disease. Value Health. (2008). Jan-Feb;, 11(1), 57-75.

[56] Lefebvre, P., Vekeman, F., Sarokhan, B., et al. Relationship between hemoglobin level and quality of life in anemic patients with chronic kidney disease receiving epoetinalfa. Curr Med Res Opin. (2006). Oct;, 22(10), 1929-1937. 
[57] Alexander, M., Kewalramani, R., Agodoa, I., et al. Association of anemia correction with health related quality of life in patients not on dialysis. Curr Med Res Opin. (2007). Dec;, 23(12), 2997-3008.

[58] Hansen, R. A., Chin, H., Blalock, S., et al. Predialysis chronic kidney disease: evaluation of quality of life in clinic patients receiving comprehensive anemia care. Res Social Adm Pharm. (2009). Jun;, 5(2), 143-153.

[59] Finkelstein, F. O., Story, K., Firanek, C., et al. Health-related quality of life and hemoglobin levels in chronic kidney disease patients. Clin J Am SocNephrol. (2009). Jan; 4(1), 33-38.

[60] Daugirdas JT, Blake PG, Ing TS. Handbook of dialysis. (3rd ed.) Philadelphia: USA: Lippincott Williams \&Wilkins: (2001).

[61] Vasilieva IA.Quality of life in chronic hemodialysis patients in Russia. Hemodial Int. (2006). , 10, 274-8.

[62] Anees, M., Hameed, F., Mumtaz, A., et al., \& Dialysis, . Dialysis-Related Factors Affecting Quality of Life in Patients on Hemodialysis. Iranian Journal of Kidney Diseases. (2011).

[63] Mollaoglu, M. Depression and health-related quality of life in hemodialysis patients. Dial. Transplant. (2004). , 544-549.

[64] Abdel-Kader, K., Unruh, M. L., Weisbord, S. D., Symptom, burden., depression, , quality, of., life, in., chronic, , end-stage, kidney., \& disease, . Clin J Am SocNephrol. (2009). Jun;, 4(6), 1057-1064.

[65] Kutner NG.Promoting functioning and well-being in older CKD patients: review of recent evidence. IntUrolNephrol. (2008). , 40(4), 1151-1158.

[66] Weisbord SD.Symptoms and their correlates in chronic kidney disease. Adv Chronic Kidney Dis. (2007). Oct;, 14(4), 319-327.

[67] Mujais, S. K., Story, K., Brouillette, J., et al. Health-related quality of life in CKD Patients: correlates and evolution over time. Clin J Am SocNephrol. (2009). Aug;, 4(8), 1293-1301.

[68] Fukuhara, S., Bito, S., Green, J., et al., Translation, adaptation., validation, of., the, S., F-, , Health, Survey., for, use., \& in, Japan. J et al. Translation, adaptation, and validation of the SF-36 Health Survey for use in Japan. J ClinEpidemiol. (1998).

[69] Germin-Petrovic, D., Mesaros-Devcić, Lesa. A., et al. Health-related Quality of Life in Hemodialysis Patients, Coll. Antropol. (2011). , 35(3), 687-693.

[70] Hunt, S. M., \& Mc Ewan, T. The development of a subjective health indicator. Soc of Health and Illness. (1980). , 231 EOF-46 EOF.

[71] Ferrans CE, Powers MJ.Quality of Life Index: development and psychometric properties. AdvNurs Sci. (1985). , 8(1), 15-24. 
[72] Jablonski, A. Level of symptom relief and the need for palliative care in the hemodialysis population. J Hospice PalliatNurs. (2007). , 9(1), 50-60.

[73] Ware JE Jr. Sherbourne CD. The MOS -item short-form health survey (SF-36).I. Conceptual framework and item selection. Med Care. (1992).

[74] Hays RD, Kallich JD, Mapes DL et al.Development of the kidney disease quality of life (KDQOL) instrument. Qual Life Res (1994).

[75] Ware Je, Snow KK, Kosinski M etal. SF-36 Health Survey: Manual Interpretation Guide Boston, MA, The Health Institute, New England Medical Center:1993.

[76] Laupacis, A., Muirhead, N., Keown, P. A., et, al. A., disease-specific, questionnaire., for, assessing., quality, of., life, in., patients, on., \& hemodialysis, . (1992). Nephron, 60(3), 302-306.

[77] Salek MS.Quality of life in patients with end-stage renal disease. J ApplTher Res. (1999). , 2(3), 163-170.

[78] Barton, A., Boyd, A., Chavez, A., et al. (2009). Health-related quality of life is maintained in hemodialysis patients receiving pharmaceutical care: A 2-year randomized, controlled study. Hemodialysis International, 13(1), 72-79.

[79] Wu AW, Fink NE, Cagney KA, et al.Developing a health-related quality of life measure for end-stage renal disease: the CHOICE Health Experience Questionnaire. Am J Kidney Dis. (2001). , 37(1), 11-21.

[80] Bradley, C. Design of a renal-dependent individualized quality of life questionnaire. AdvPerit Dial. (1997). , 13, 116-120.

[81] Barthel, D., Functional, evaluation., the, Barthel., \& Index, . J. (1965). , 14, 61-65.

[82] Granger CV, Devis LS, Peters MC, et al. Stroke rehabilitation: analysis of repeated Barthel Index measures.Arch Phys Med Rehabil. (1979). , 60, 14-17.

[83] Melzack, R. The McGill Pain Questionnaire: Major properties and scoring methods. Pain. (1975). , 1, 277-299.

[84] Draper, P. Quality of life as quality of being: An alternative to subject-object dichotomy. J A. d Nurs. 992; , 17, 965-970.

[85] Drueke, T. B., Locatelli, F., Clyne, N., et al. Normalization of hemoglobin level in patients with chronic kidney disease and anemia. N Engl J Med. (2006). Nov 16; 355(20), 2071-2084.

[86] Singh, A. K., Szczech, L., Tang, K. L., et al. Correction of anemia with epoetinalfa in chronic kidney disease. N Engl J Med. (2006). Nov 16;, 355(20), 2085-2098.

[87] KDOQI Clinical Practice Guideline and Clinical Practice Recommendations for anemia in chronic kidney disease:(2007). update of hemoglobin target. Am J Kidney Dis. 2007 Sep; , 50(3), 471-530. 
[88] Feldt-Rasmussen, B., Lange, M., Sulowicz, W., et al. Growth hormone treatment during hemodialysis in a randomized trial improves nutrition, quality of life, and cardiovascular risk. J Am SocNephrol. (2007). Jul;, 18(7), 2161-2171.

[89] White, Y. ., \& Grenyer, B. F. S. (1999). The biopsychosocial impact of end-stage renal disease: the experience of dialysis patients and their partners. J AdvNurs. 30(6), , 1312 EOF.

[90] Parkerson GR, Gutman RA.Health-related quality of life predictors of survival and hospital utilization. Health Care Financ R. (2000). , 21(3), 171-185.

[91] Tsay SL, Lee YC.Effects of an adaptation training programme for patients with endstage renal disease. J AdvNurs (2005). , 50, 39-46.

[92] Chang CF, WinsettRP,Gaber AO, et al.Cost-effectiveness of post-transplantation quality of life intervention among kidney recipients. ClinTranspl. (2004). , 18, 407-414.

[93] Gross, C. R., Kreitzer, Russas. V., et al. Mindfulness meditation to reduce symptoms after organ transplant: a pilot study. Adv Mind Body Med. (2004). , 20, 20-29. 
